same day discharge. Patients were excluded if they had any of the following: an unsuitable radial pulse, planned femoral access, prior coronary artery bypass surgery, or the requirement for an overnight hospital stay for planned complex/high risk PCI, renal impairment, or social reasons. The final decision regarding route of arterial access was left to the operator.

Results In the one year study period, 1548 patients were managed in the radial lounge. 1109 patients underwent coronary angiography, $114(10.2 \%)$ of whom also had a pressure wire or intravascular ultrasound, and 439 underwent PCI. This represented approximately $88 \%$ of our unit's elective angiograms and $60 \%$ of our elective PCIs. Among the patients who underwent angiography, 938 (84.5\%) were performed radially and 1076 (97.0\%) were discharged from the radial lounge on the same day as their procedure. Among the PCI patients, 359 (81.8\%) were performed radially and 372 $(84.7 \%)$ were discharged the same day. The PCI group included 326 $(74.3 \%)$ patients who had a single vessel treated, 105 (23.9\%) who had two vessels or a bifurcation with a significant side branch treated, and $8(1.8 \%)$ patients who had three vessels treated. There were no deaths or arrhythmias in the radial lounge. Requirement for overnight admission was significantly more common after femoral access compared with radial access for both angiography (4.1\% vs $2.8 \% ; \mathrm{p}<0.05)$ and PCI (21.3\% vs $14.2 \% ; \mathrm{p}<0.01)$

Conclusions A dedicated radial lounge free of cardiac monitors is a safe environment in which to manage most patients before and after elective coronary angiography and PCI. The lack of monitoring necessitates patient selection but this does not prevent the lounge being suitable for the majority of elective patients. Femoral access is associated with a significantly greater requirement for overnight admission.

\section{REMAINING CLOTHED FOR RADIAL DIAGNOSTIC CORONARY ANGIOGRAPHY: AN IMPROVEMENT IN THE PATIENT JOURNEY}

doi:10.1136/heartjnl-2011-300198.79

S Eve, M Sinha, T A Wells. Salisbury District Hospital, Salisbury, UK

Background Patients undergoing invasive diagnostic coronary angiography (DCA) for the first time often display high levels of anxiety at the time of their procedure as they are unfamiliar with the cardiac catheter laboratory set up. It is therefore part of the cardiac catheter laboratory staff's role to reduce patient fears and hence improve their journey through the cardiac catheter laboratory. Several Cardiac centres have recently introduced radial lounges whereby patients feel less "hospitalised" by not needing to undress for their procedure.

Methods Following infection control approval, between mid-August 2010 and the end of October 2010, patients undergoing radial DCA at Salisbury District Hospital were offered the option of remaining clothed for their procedure. Each patient was given an information leaflet included in which was explained possible downsides to being dressed including if CPR were needed then clothes would be cut, failure of radial access and the subsequent need for femoral access, and the possibility of soiling the clothes with either blood or iodine. The only caveat stated was that female patients were not allowed to wear an underwire bra. Following their DCA, patients were then asked to fill in an anonymous questionnaire in which they were asked about their experience and whether not having to undress made them feel more relaxed.

Results 57 consecutive patients underwent (DCA) during this time period $(100 \%$ uptake) with an average age of $68.1 \pm 9.6$ years. $71 \%$ were male and $21 \%(12 / 57)$ had undergone a DCA previously. Of these 12 patients $92 \%(11 / 12)$ stated that not having to undress was a good idea while an identical number felt much more relaxed than their previous DCA experience. Of the 45 patients that had not had a previous DCA, $96 \%(43 / 45)$ stated that not having to undress was a good idea while $96 \%$ (43/45) felt that this had made them feel very relaxed during their pathway. The other two patients felt that it made no difference. No patients required cross-over to femoral access and there were no blood or iodine stains on any clothes. Having patients remain dressed did not reduce fluoroscopic image quality and there were no issues with infection.

Conclusion Offering patients the option of having their radial DCA done without undressing is safe and helps to improve the patient journey through the cardiac catheter laboratory by making them feel more relaxed and less hospitalised. This is now standard at our Institution.

\section{PPCI: IS THERE A ROLE FOR THE ACS ANP?}

doi:10.1136/heartjnl-2011-300198.80

V Oriolo, J Tagney. Bristol Heart Institute University Hospital Bristol NHS FT, Bristol, UK

Introduction Primary Percutaneous Coronary Intervention (PPCI) is now considered the treatment of choice for patients experiencing ST Elevation Myocardial Infarction (STEMI) (European Society of Cardiology/European Association for Cardio-Thoracic Surgery 2010). One of the many benefits claimed is reduced length of stay due to decreased morbidity (Zahn et al 2000, Kalla et al 2006). To assess performance of one English PPCI 24/7 provider organisation against the national average length of stay (LOS) for patients post PPCI, a retrospective baseline audit was conducted. This demonstrated an average LOS of 4.4 days which is above the National Infarct Angioplasty Project 2008 average LOS of 3 days. A 48 h nurse led discharge (NLD) protocol was therefore developed and introduced by the acute coronary syndromes advanced nurse practitioner to streamline the patient journey. This instructed the nurse and/or physician to ensure appropriate investigation and documentation was carried out in a timely manner to avoid unnecessary delays in patient discharge.

Method Suitability criteria for the 48-h NLD protocol were established, which included: absence of acute complications (eg, bleeding, haemodynamic instability, ongoing chest pain, ejection fraction $<40 \%$, respiratory compromise); appropriate support at discharge. Following the baseline audit, data were electronically collected prospectively for 5 months, measuring date of admission to date of discharge to the usual place of residence. After 5 months the audit was repeated to assess the average length of stay for patients presenting with STEMI.

Result Between 1st April 2010 and 31st August 2010, a total of 274 patients were admitted with STEMI. Of these, 122 (45\%) met the NLD criteria and were discharged by the ACS ANP. The remaining $152(55 \%)$ were discharged by the medical physicians. It was noted that introduction of the protocol also facilitated a structured approach to discharge for the medical team. The average LOS for all PPCI patients $(n=274)$ decreased from 4.4 days to 3 days $(30 \%)$. For patients that were seen and discharged solely by the advanced nurse practitioner $(n=122)$, the average LOS decreased from 4.4 days to 2.0 days $(55 \%)$

Conclusions/Implications In the current financial climate, a decrease in LOS can have a significant impact on any organisational resources thus increasing efficiency saving and patient throughput. This demonstrates part of the added value the advanced nurse practitioner brings to patient care and to tertiary centres that provide a 24/7 PPCI service. 